\title{
Association of circulating PCSK9 concentration with cardiovascular metabolic markers and outcomes in stable coronary artery disease patients with or without diabetes: a prospective, observational cohort study
}

Jia Peng ${ }^{\dagger}$, Ming-Ming Liu ${ }^{\dagger}$, Jing-Lu Jin, Ye-Xuan Cao, Yuan-Lin Guo, Na-Qiong Wu, Cheng-Gang Zhu, Qian Dong, Jing Sun, Rui-Xia Xu* and Jian-Jun Li ${ }^{*}$ (1)

\begin{abstract}
Background: Whether plasma proprotein convertase subtilisin/kexin type 9 (PCSK9) levels is a predictor for cardiovascular outcomes has currently been controversial. No data is currently available regarding the relation of PCSK9 to cardiovascular metabolic markers (CVMMs) and major adverse cardiovascular events (MACEs) in stable coronary artery disease (CAD) patients with diabetes or without diabetes.

Methods: A total 1225 untreated patients with stable CAD were consecutively enrolled and their baseline plasma PCSK9 levels were determined by ELISA. Patients were divided into high and low PCSK9 groups according to PCSK9 median. All patients followed up for the occurrence of MACEs and received standard therapy after admission. The associations of PCSK9 with CVMMs and MACEs were evaluated.

Results: PCSK9 levels were positively correlated with multiple CVMMs including total cholesterol, low-density lipoprotein cholesterol, non-high-density lipoprotein cholesterol and hemoglobin $A_{1 c}$ at baseline (all $p<0.05$ ). During a median follow-up of 3.3 years, 103 (8.4\%) events occurred. PCSK9 levels were higher in patients with events compared to those without $(p<0.05)$. The Kaplan-Meier analysis displayed that patients in high PCSK9 group had lower eventfree survival than that in low group $(p<0.05)$. Multivariable Cox regression analysis revealed that PCSK9 levels were independently associated with MACEs in diabetic patients (adjusted hazard ratio [HR]: 1.361, 95\% confidence interval [CI]: 1.037-1.785, $p<0.05)$. When added the combination of PCSK9 levels and diabetic status to stratifying factors, patients in high PCSK9 group appeared to have extremely high risk of subsequent MACEs with diabetes (adjusted HR: $5.233,95 \%$ Cl: $2.546-10.757, p<0.01)$.
\end{abstract}

*Correspondence: ruixiaxu@sina.com; lijianjun938@126.com

${ }^{\dagger} \mathrm{Jia}$ Peng and Ming-Ming Liu contribute equally to the article.

State Key Laboratory of Cardiovascular Diseases, Fu Wai Hospital, National

Center for Cardiovascular Diseases, Chinese Academy of Medical Sciences

and Peking Union Medical College, No 167 BeiLiShi Road, XiCheng

District, Beijing 100037, China

(c) The Author(s) 2020. This article is licensed under a Creative Commons Attribution 4.0 International License, which permits use, sharing, adaptation, distribution and reproduction in any medium or format, as long as you give appropriate credit to the original author(s) and the source, provide a link to the Creative Commons licence, and indicate if changes were made. The images or other third party material in this article are included in the article's Creative Commons licence, unless indicated otherwise in a credit line to the material. If material is not included in the article's Creative Commons licence and your intended use is not permitted by statutory regulation or exceeds the permitted use, you will need to obtain permission directly from the copyright holder. To view a copy of this licence, visit http://creativecommons.org/licenses/by/4.0/. The Creative Commons Public Domain Dedication waiver (http://creativecommons.org/publicdomain/zero/1.0/) applies to the data made available in this article, unless otherwise stated in a credit line to the data. 
Conclusions: The present study firstly showed that elevated PCSK9 levels were related to multiple CVMMs and MACEs in stable CAD with diabetes, suggesting that plasma PCSK9 measurement could help to identify diabetic patients with CAD at higher cardiovascular risk. More studies may be needed to confirm our findings.

Keywords: PCSK9, DM, CAD, Maces, Cardiovascular metabolic markers

\section{Background}

Diabetes mellites (DM) is regarded as coronary artery disease (CAD) equivalent and associated with an obvious increase in the risk of atherosclerotic cardiovascular disease (ASCVD) [1, 2]. Meanwhile, ASCVD-related mortality has been reported to be significantly higher in diabetic patients than that in nondiabetic patients (20 vs 3.5\%) [3]. Additionally, DM is commonly accompanied with multifaceted metabolic disorders and the risk of ASCVD in patients with DM would be amplified by several concurrent atherogenic metabolic factors [4]. More importantly, atherogenic dyslipidemia is one of the major common clinical manifestations, which is an important predictive factor of cardiovascular risks in diabetic individuals. The management of this type of dyslipidemia, especially targeting low-density lipoprotein cholesterol (LDL-C) levels with optimal strategies, can significantly bring cardiovascular benefits in diabetic patients [5-7].

Proprotein convertase subtilisin/kexin type 9 (PCSK9), a serine protease belonging to the proteinase $\mathrm{K}$ subfamily of subtilases, is a critical player in cholesterol metabolism by increasing the degradation of hepatic low-density lipoprotein cholesterol receptors (LDLR), thereby resulting in increasing circulating LDL-C levels $[8,9]$. Recently, a large number of studies have demonstrated that PCSK9 can directly or indirectly contribute to atherosclerosis from initiation to progression by leading to endothelial dysfunction, promoting inflammatory response and inhibiting platelet activation, which is independent on its effects on regulation of cholesterol metabolism [10-12]. Consequently, several large-scale event reduction trials have found that human PCSK9 monoclonal antibodies markedly reduce LDL-C levels and show a great promise for a reduction of future cardiovascular events [13, 14]. It is worth noting that circulating PCSK9 concentration has been proposed as an adverse factor for cardiovascular risks in patients with CAD $[15,16]$. What's more, a growing number of studies have explored the association of PCSK9 with cardiovascular metabolic disorders beyond dyslipidemia [17-19]. Nevertheless, the study on the association between plasma PCSK9 levels and cardiovascular metabolic markers (CVMMs) is limited in diabetic patients with CAD and whether plasma PCSK9 concentration can predict major adverse cardiovascular events (MACEs) in these patients has not been evaluated.

Hence, in the current study, we try to investigate the association of plasma PCSK9 levels at baseline with CVMMs and the future MACEs in stable CAD patients with DM or without DM, who did not take any lipid-lowering therapy before admission and received a standard treatment after admission.

\section{Method \\ Study population}

The present study protocol was complied with Declaration of Helsinki and was approved by the hospital ethics review board (Fu Wai Hospital \& National Center for Cardiovascular Diseases, Beijing, China, approval number: 2013-442). Every patient signed informed written consent before enrolled in this study.

As shown in the flowchart (Fig. 1), from October 2012 to March 2018, 2259 patients with first angina-like chest pain, who took no lipid-lowering drugs within 3 months before admission were enrolled in the current study. All of them completed coronary angiography and/or coronary computed tomography to confirm their diagnosis of CAD. Among them, 949 patients without CAD (coronary stenosis $\geq 50 \%$ of at least one coronary artery) were excluded. Additionally, patients diagnosed as acute coronary syndrome or previous myocardial infarction (MI), patients without PCSK9 or detailed laboratory data, patients with familial hypercholesterolemia (FH), severe infection, severe liver or renal insufficiency (plasma creatinine $<150 \mu \mathrm{mol} / \mathrm{L}$ at baseline), hematology disorders, thyroid dysfunction and malignant tumor were also excluded from this study. Finally, 1288 patients firstly diagnosed as stable CAD were included in this cohort and received standard treatments after admission and followed up.

\section{Blood sample measurement}

Blood samples of all participants were collected after a 10-12 h overnight fasting immediately after their admission. Plasma samples were prepared by centrifugation at $3500 \times g$ twice for $10 \mathrm{~min}$ each at $15-18{ }^{\circ} \mathrm{C}$ and were stored at $-80^{\circ} \mathrm{C}$ until analysis according to our previous studies [20-22].

Plasma PCSK9 levels were measured using a highsensitivity, quantitative sandwich enzyme immunoassay 
2259 hospitalized patients who had first angina-chest pain and received coronary angiography or coronary computed tomography without lipid-lowering therapy from October 2012 to March 2018

1419 patients with stable CAD

949 non-CAD patients

25 patients with acute coronary syndrome

63 patients lost follow-up

131 patients with missing data, familial hypercholesterolemia, severe infectious, severe liver or renal insufficiency hematology disorders, thyroid dysfunction and malignant tumor

1225 patients for analysis

Fig. 1 Flowchart of the study. CAD coronary artery disease, non-DM non-diabetes mellitus, DM diabetes mellitus

(Quantikine ELISA, R\&D Systems Europe Ltd) according to our previous study [22]. The lower limit of detection was $0.096 \mathrm{ng} / \mathrm{mL}$. PCSK9 levels of each patient were determined twice and the mean value of the two samples was used in the final analysis. Plasma levels of fibrinogen were quantitatively measured by the method of Clauss and a Stago autoanalyzer with STA Fibrinogen kit (Diagnostic Stago, Taverny, France). The levels of plasma triglyceride (TG), total cholesterol (TC), high-density lipoprotein cholesterol (HDL-C), LDL-C, apolipoprotein (apo) A1, apoB and fasting plasma glucose (FPG) were determined by automatic biochemistry analyser (Hitachi 7150, Japan). Hemoglobin $\mathrm{A}_{1 \mathrm{C}}\left(\mathrm{HbA}_{1 \mathrm{C}}\right)$ was determined using Tosoh Automated Glycohemoglobin Analyser (HLC-723G8, Tokyo, Japan).

\section{Follow up}

Patients were followed up by telephone and/or clinic revisit every six months, which was conducted by welltrained doctors or nurses. They were blinded to the 
clinical data of these patients and the purpose of this study until death occurred or the last day of followup period. Finally, 63 patients were lost to follow-up, the resulting population consisted of 1225 stable CAD patients taking no lipid-lowering medicines before admission. The following MACEs included hospitalization for unstable angina, coronary revascularization, nonfatal MI, ischemic stroke, and cardiovascular death.

\section{Diagnosis of clinical disease}

DM was considered present if patients met one of the following criteria: self-reported DM, FPG levels $\geq 7.0 \mathrm{mmol} / \mathrm{L}(126 \mathrm{mg} / \mathrm{dL})$, fasting plasma $\mathrm{HbA}_{1 \mathrm{C}}$ levels $\geq 6.5 \%$, or treated with oral hypoglycemic agents or insulin. Hypertension was defined as systolic blood pressure $(\mathrm{BP}) \geq 140 \mathrm{mmHg}$ or diastolic $\mathrm{BP} \geq 90 \mathrm{mmHg}$ or the use of anti-hypertensive drugs. Information regarding other diseases, family history and prior therapy of every patient was collected from self-reported medical history. Unstable angina was confirmed if patients appeared rest angina or new-onset severe angina with normal serum levels of cardiac enzymes that required admission. Coronary revascularization included percutaneous coronary intervention (PCI) and coronary artery bypass grafting (CABG) later than 90 days after their discharge. Nonfatal MI was diagnosed as typical angina-like chest pain with increased cardiac troponins or typical electrocardiogram changes. Ischemic stroke was described by acute cerebral infarction symptoms and cerebral diagnostic imaging.

\section{Calculation of parameters}

Body mass index (BMI) was determined as body weight $(\mathrm{kg})$ divided by body height $\left(\mathrm{m}^{2}\right)$. Non-high-density lipoprotein cholesterol (non-HDL-C) was calculated as TC minus HDL-C, triglyceride-rich lipoprotein was calculated as non-HDL-C minus LDL-C as the previous study showed [23]. The value of atherogenic index of plasma was defined as the base 10 logarithm of the ratio of the concentration of TG to HDL-C, the lipoprotein combine index (LCI) was defined as the ratio of TC $\times$ TG $\times$ LDL-C to HDL-C [24]. Triglyceride glucose was calculated as Ln [fasting TG $(\mathrm{mg} / \mathrm{dL}) \times$ FPG $(\mathrm{mg} / \mathrm{dL}) / 2$ ], hemoglobin glycation index (HGI) was calculated as subtracting the predicted $\mathrm{HbA}_{1 \mathrm{C}}$ from the observed $\mathrm{HbA}_{1 \mathrm{C}}[25,26]$. The predicted $\mathrm{HbA}_{1 \mathrm{C}}$ was calculated for all participants by inserting the baseline FPG into the subsample linear regression equation derived from another randomly selected 942 patients who admitted to our division $\left[\mathrm{HbA}_{1 \mathrm{C}}=0.113 \times \mathrm{FPG}(\mathrm{mg} / \mathrm{dL})+5.289\right]$.

\section{Evaluation of coronary severity}

The Gensini score was used to evaluate the severity of coronary stenosis and was calculated as the location score for all diseased segments multiplied by the stenosis score [27], which was performed by two experienced interventional cardiologists according to our previous studies [21, 22].

\section{Statistical analysis}

Continuous variables were expressed as mean \pm standard deviation (SD) or median (25th-75th percentile). Categorical variables were expressed as frequencies (percentage). Student's $t$ test or Mann Whitney U-test were used to compare the statistic difference of continuous variables between two groups. $X^{2}$ analysis and Fisher's test were performed to determine the statistic difference in categorical variables between the two groups. Spearman correlation analysis was used to evaluate the correlation with plasma PCSK9 levels and CVMMs. The cumulative event-free survival rates of MACEs among subgroups according to PCSK9 levels or/and diabetic status were examined by the Kaplan-Meier curve with the log-rank test. Univariate and multivariate Cox regression analyses were applied to calculate the hazard ratios (HR) of incident MACEs with $95 \%$ confidence intervals (CI). A $p$ value $<0.05$ was considered to be statistically difference. Statistical analysis was performed with Statistical Package for Social Sciences version 25.0.

\section{Results}

\section{Baseline characteristics}

During the follow-up period, 1225 patients firstly diagnosed as stable CAD were enrolled in the current study. The mean age of this population was $57.8 \pm 10.1$ years, 833 (68\%) participants were male, and 377 (30.8\%) patients suffered DM. The interquartile PCSK9 levels ranged from 194.79 to $276.13 \mathrm{ng} / \mathrm{mL}$, with a median of $234.52 \mathrm{ng} / \mathrm{mL}$. The baseline characteristics of the cohort were shown in Table 1 according to event occurrence during follow-up. Patients with events had clinical features presented as higher TC, LDL-C, FPG, $\mathrm{HbA}_{1 \mathrm{C}}$ and fibrinogen levels, higher percentage of hypertension, $\mathrm{DM}$ and family history of CAD, higher Gensini score and tend to have more multi-diseased coronary vessels (all $p<0.05)$. However, there were no significant differences with regard to the percentage of gender, current smoker and drinker, TG and HDL-C levels, left ventricle ejection fraction and medication prescriptions (except $\beta$-blockers) in subjects with and without events. Notably, circulating PCSK9 concentration at baseline was significantly higher in patients with events than that in counterparts [264.26 (219.45, 309.86) vs 232.99 (193.72, 274.16) ng/ $\mathrm{mL}, p<0.001]$. Meanwhile, we further analyzed the postadmission therapy in all patients and found that there were no significant differences in coronary revascularization including PCI and CABG and medications including 
Table 1 Baseline characteristics in study patients with and without events

\begin{tabular}{|c|c|c|c|c|}
\hline & Total $(n=1225)$ & Non-events $(n=1122)$ & Events $(n=103)$ & $p$ value \\
\hline \multicolumn{5}{|l|}{ Clinical characteristics } \\
\hline Age (years) & $57.8 \pm 10.1$ & $57.7 \pm 10.0$ & $58.5 \pm 10.8$ & 0.419 \\
\hline Male sex & $833(68.0)$ & $765(68.3)$ & $68(66.0)$ & 0.653 \\
\hline $\mathrm{BMI}\left(\mathrm{kg} / \mathrm{m}^{2}\right)$ & $25.9 \pm 3.3$ & $25.9 \pm 3.2$ & $26.0 \pm 4.1$ & 0.704 \\
\hline Hypertension & $854(69.7)$ & $781(69.6)$ & $73(70.9)$ & 0.789 \\
\hline Family history of CAD & $289(23.6)$ & $275(24.5)$ & $14(13.6)$ & 0.013 \\
\hline Diabetes mellites & $377(30.8)$ & $327(29.1)$ & $50(48.5)$ & $<0.001$ \\
\hline Current smoker & $481(39.3)$ & $440(39.2)$ & $41(39.8)$ & 0.907 \\
\hline Drinking & $284(23.2)$ & $261(23.3)$ & $23(22.3)$ & 0.830 \\
\hline \multicolumn{5}{|l|}{ Laboratory parameters } \\
\hline FPG (mmol/L) & $5.9 \pm 1.9$ & $5.9 \pm 1.8$ & $6.3 \pm 3.1$ & 0.042 \\
\hline $\mathrm{HbA1C}(\%)$ & $6.2 \pm 1.1$ & $6.2 \pm 1.1$ & $6.4 \pm 1.1$ & 0.036 \\
\hline $\mathrm{ALT}(\mathrm{U} / \mathrm{L})$ & $20(15,29)$ & $20(15,29)$ & $20(14,31)$ & 0.905 \\
\hline Creatinine $(\mu \mathrm{mol} / \mathrm{L})$ & $77.7 \pm 17.5$ & $77.6 \pm 16.9$ & $79.1 \pm 23.0$ & 0.408 \\
\hline $\mathrm{TC}(\mathrm{mmol} / \mathrm{L})$ & $4.82 \pm 0.96$ & $4.80 \pm 0.95$ & $5.02 \pm 0.95$ & 0.028 \\
\hline $\mathrm{TG}(\mathrm{mmol} / \mathrm{L})$ & $1.61(1.16,2.32)$ & $1.6(1.15,2.31)$ & $1.72(1.31,2.43)$ & 0.215 \\
\hline $\mathrm{HDL}-\mathrm{C}(\mathrm{mmol} / \mathrm{L})$ & $1.09 \pm 0.32$ & $1.09 \pm 0.32$ & $1.15 \pm 0.38$ & 0.060 \\
\hline LDL-C (mmol/L) & $3.15 \pm 0.85$ & $3.13 \pm 0.85$ & $3.35 \pm 0.85$ & 0.013 \\
\hline PCSK9 (ng/mL) & $234.52(194.79,276.13)$ & $232.99(193.72,274.16)$ & $264.26(219.45,309.86)$ & $<0.001$ \\
\hline Fibrinogen $(\mu \mathrm{g} / \mathrm{mL})$ & $3.1 \pm 0.8$ & $3.1 \pm 0.7$ & $3.3 \pm 1.1$ & 0.006 \\
\hline NT-proBNP (pg/mL) & $51.9(31.0,109.3)$ & $50.9(30.1,111.0)$ & $58.1(38.1,96.7)$ & 0.172 \\
\hline \multicolumn{5}{|l|}{ Diseased vessels } \\
\hline One vessel & $438(35.8)$ & $411(36.6)$ & $27(26.2)$ & \\
\hline Two vessels & $365(29.8)$ & $340(30.3)$ & $25(24.3)$ & \\
\hline Three vessels & $422(34.4)$ & $371(33.1)$ & $51(49.5)$ & \\
\hline LVEF (\%) & $64.7 \pm 6.7$ & $64.7 \pm 6.7$ & $64.8 \pm 6.6$ & 0.882 \\
\hline Gensini score & $27(11,44)$ & $26(10,42)$ & $35.08(18,60)$ & 0.003 \\
\hline \multicolumn{5}{|l|}{ Medications } \\
\hline Aspirin & $464(37.9)$ & $431(38.4)$ & $33(32.0)$ & 0.202 \\
\hline Clopidogrel & $130(10.6)$ & $123(11.0)$ & $7(6.8)$ & 0.189 \\
\hline ACEI/ARB & $252(20.6)$ & $235(20.9)$ & $17(16.5)$ & 0.286 \\
\hline$\beta$-blockers & $238(19.4)$ & $226(20.1)$ & $12(11.7)$ & 0.037 \\
\hline
\end{tabular}

PCSK9 proprotein convertase subtilisin/kexin type 9, BMI body mass index, CAD coronary artery disease, FPG fasting plasma glucose, $H b A_{1 C}$ hemoglobin $\mathrm{A}_{1 C}, A L T$ alanine aminotransferase, $T C$ total cholesterol, $T G$ triglyceride, $H D L-C$ high-density lipoprotein cholesterol, $L D L-C$ low-density lipoprotein cholesterol, $N T$-proBNP $\mathrm{N}$-Terminal pro-brain natriuretic peptide, $L V E F$ left ventricular ejection fraction, $A C E$ langiotensin converting enzyme inhibitors, $A R B$ angiotensin receptor blockers, $p<0.05$ suggests significant difference

lipid-lowering drugs between patients with events and without events (all $p>0.05)$.

Next, we divided the entire study population into two groups according to the median of PCSK9 levels at baseline (shown in Table 2). Patients in low PCSK9 group were younger, female, and more likely to be current smoker and drinker (all $p<0.05$ ). Furthermore, baseline levels of plasma TC, LDL-C, HDL-C, FPG, $\mathrm{HbA}_{1 \mathrm{C}}$ and fibrinogen were significantly higher in participants with high PCSK9 levels compared to those with low PCSK9 levels (all $p<0.05$ ). Besides, BMI, percentage of hypertension and DM, TG levels, Gensini score and medication use were no significant differences between two groups (all $p<0.05)$. Additionally, as presented in Additional file 1: Table S1, we also found that CAD subjects with DM had higher PCSK9, TG levels and Gensini score, lower HDL-C levels, and more likely to occur multidiseased coronary vessels compared with nondiabetic patients (all $p<0.05)$.

\section{Association of PCSK9 with CVMMs}

Spearman correlation analysis showed that plasma PCSK9 levels were significantly and positively associated with multiple CVMMs, especially with glycemic 
Table 2 Baseline characteristics of patients according to PCSK9 stratification

\begin{tabular}{|c|c|c|c|c|}
\hline \multirow[t]{2}{*}{ Variables } & \multirow[t]{2}{*}{ Total $(n=1225)$} & \multicolumn{2}{|c|}{ PCSK9 concentration $(\mathrm{ng} / \mathrm{mL})$} & \multirow[t]{2}{*}{$p$ value } \\
\hline & & $<234.52(n=612)$ & $\geq 234.52(n=613)$ & \\
\hline \multicolumn{5}{|l|}{ Clinical characteristics } \\
\hline Age (years) & $57.8 \pm 10.1$ & $57.1 \pm 10.1$ & $58.4 \pm 10.0$ & 0.027 \\
\hline Male sex & $833(68.0)$ & $469(76.6)$ & $364(59.4)$ & $<0.001$ \\
\hline $\mathrm{BMI}\left(\mathrm{kg} / \mathrm{m}^{2}\right)$ & $25.9 \pm 3.3$ & $26.0 \pm 3.5$ & $25.8 \pm 3.2$ & 0.217 \\
\hline Hypertension & $854(69.7)$ & $424(69.3)$ & $430(70.1)$ & 0.742 \\
\hline Family history of $C A D$ & $289(23.6)$ & $126(20.6)$ & $163(26.6)$ & 0.013 \\
\hline Diabetes mellites & $377(30.8)$ & $180(29.4)$ & $197(32.1)$ & 0.301 \\
\hline Current smoker & $481(39.3)$ & $258(53.6)$ & $223(46.4)$ & 0.038 \\
\hline Drinking & $284(23.2)$ & $159(56.0)$ & $125(44.0)$ & 0.020 \\
\hline \multicolumn{5}{|l|}{ Laboratory parameters } \\
\hline $\mathrm{FPG}(\mathrm{mmol} / \mathrm{L})$ & $5.9 \pm 1.9$ & $5.7 \pm 1.7$ & $6.0 \pm 2.1$ & 0.012 \\
\hline $\mathrm{HbA}_{1 \mathrm{C}}(\%)$ & $6.2 \pm 1.1$ & $6.1 \pm 1.0$ & $6.3 \pm 1.2$ & 0.010 \\
\hline $\mathrm{ALT}(\mathrm{U} / \mathrm{L})$ & $20(15,29)$ & $20(15,28)$ & $20(14,30)$ & 0.769 \\
\hline Creatinine ( $\mu \mathrm{mol} / \mathrm{L})$ & $77.7 \pm 17.5$ & $77.9 \pm 16.1$ & $77.5 \pm 18.8$ & 0.680 \\
\hline $\mathrm{TC}(\mathrm{mmol} / \mathrm{L})$ & $4.82 \pm 0.96$ & $4.67 \pm 0.90$ & $4.98 \pm 0.99$ & $<0.001$ \\
\hline $\mathrm{TG}(\mathrm{mmol} / \mathrm{L})$ & $1.61(1.16,2.31)$ & $1.55(1.12,2.30)$ & $1.65(1.20,2.34)$ & 0.226 \\
\hline $\mathrm{HDL}-\mathrm{C}(\mathrm{mmol} / \mathrm{L})$ & $1.09 \pm 0.32$ & $1.07 \pm 0.33$ & $1.12 \pm 0.32$ & 0.011 \\
\hline $\mathrm{LDL}-\mathrm{C}(\mathrm{mmol} / \mathrm{L})$ & $3.15 \pm 0.85$ & $3.00 \pm 0.78$ & $3.29 \pm 0.89$ & $<0.001$ \\
\hline Fibrinogen $(\mu \mathrm{g} / \mathrm{mL})$ & $3.1 \pm 0.8$ & $3.0 \pm 0.6$ & $3.2 \pm 0.8$ & $<0.001$ \\
\hline NT-proBNP (pg/mL) & $51.9(31.0,109.3)$ & $49.45(31.2,92.5)$ & $53.2(30.4,130.0)$ & 0.083 \\
\hline \multicolumn{5}{|l|}{ Diseased vessels } \\
\hline One vessel & $438(35.8)$ & $241(39.4)$ & $197(32.1)$ & \\
\hline Two vessels & $365(29.8)$ & $173(28.2)$ & $192(31.3)$ & \\
\hline Three vessels & $422(34.4)$ & $198(32.4)$ & $224(36.5)$ & \\
\hline $\operatorname{LVEF}(\%)$ & $64.7 \pm 6.7$ & $64.6 \pm 6.9$ & $64.8 \pm 6.6$ & 0.716 \\
\hline Gensini score & $27(11,44)$ & $25(10,43)$ & $28(12,44)$ & 0.095 \\
\hline \multicolumn{5}{|l|}{ Medications } \\
\hline Aspirin & $464(37.9)$ & $228(37.3)$ & $236(38.5)$ & 0.654 \\
\hline Clopidogrel & $130(10.6)$ & $61(10.0)$ & $69(11.3)$ & 0.464 \\
\hline ACEI/ARB & $252(20.6)$ & $119(19.4)$ & $133(21.7)$ & 0.330 \\
\hline$\beta$-blockers & $238(19.4)$ & $108(17.6)$ & $130(21.2)$ & 0.115 \\
\hline MACES & $103(8.4)$ & $36(5.9)$ & $67(10.9)$ & 0.001 \\
\hline
\end{tabular}

PCSK9 proprotein convertase subtilisin/kexin type 9, $B M /$ body mass index, $C A D$ coronary artery disease, $F P G$ fasting plasma glucose, $H b A_{1 C}$ hemoglobin $A_{1 C}, A L T$ alanine aminotransferase, $T C$ total cholesterol, $T G$ triglyceride, $H D L-C$ high-density lipoprotein cholesterol, $L D L-C$ low-density lipoprotein cholesterol, $N T$-proBNP $\mathrm{N}$-Terminal pro-brain natriuretic peptide, $L V E F$ left ventricular ejection fraction, $A C E I$ angiotensin converting enzyme inhibitors, $A R B$ angiotensin receptor blockers, MACEs major adverse cardiovascular events, $p<0.05$ suggests significant difference

and lipid parameters including traditional, novel and derived lipid indicators (shown in Table 3) in CAD patients. Furthermore, our results found a significantly positive correlation of PCSK9 with novel derived CVMMs including LCI and HGI in stable CAD patients ( $\mathrm{r}=0.112, p<0.0001 ; \mathrm{r}=0.112, p<0.0001$, respectively). Meanwhile, the further analysis suggested that positive relationships between PCSK9 and several lipid-related markers including TC, LDL-C, non-HDL-C, apoA1 and apoB remained in DM group $(r=0.133, p=0.010$; $\mathrm{r}=0.153, \quad p=0.003 ; \quad \mathrm{r}=0.104, \quad p=0.043 ; \quad \mathrm{r}=0.104$, $p=0.044 ; \mathrm{r}=0.138, p=0.007$, respectively) and nonDM group $(\mathrm{r}=0.234, p<0.0001 ; \mathrm{r}=0.215, p<0.0001$; $\mathrm{r}=0.217, \quad p<0.0001 ; \mathrm{r}=0.090, \quad p=0.009 ; \mathrm{r}=0.213$, $p<0.0001$, respectively). Additionally, PCSK9 levels were positively associated with $\mathrm{HbA}_{1 \mathrm{C}}$ in $\mathrm{DM}$ patients $(\mathrm{r}=0.111, p=0.032)$, which was consistent with our previous study [18]. A weak correlation of PCSK9 and $\mathrm{HbA}_{1 \mathrm{C}}$ was also observed in non-DM group $(\mathrm{r}=0.098$, $p=0.004$ ). 
Table 3 Spearman correlation analysis between PCSK9 and cardiovascular metabolic makers

\begin{tabular}{|c|c|c|c|c|c|c|}
\hline \multirow[t]{3}{*}{ Variables } & \multirow{2}{*}{\multicolumn{2}{|c|}{$\begin{array}{l}\text { Overall } \\
n=1225\end{array}$}} & \multirow{2}{*}{\multicolumn{2}{|c|}{$\begin{array}{l}\mathrm{DM} \\
\mathrm{n}=377\end{array}$}} & \multirow{2}{*}{\multicolumn{2}{|c|}{$\begin{array}{l}\text { Non-DM } \\
\mathrm{n}=848\end{array}$}} \\
\hline & & & & & & \\
\hline & $r$ & $p$ & $r$ & $p$ & $r$ & $p$ \\
\hline \multicolumn{7}{|c|}{ Traditional lipid parameters } \\
\hline $\mathrm{TC}$ & 0.199 & $<0.0001$ & 0.133 & 0.010 & 0.234 & $<0.0001$ \\
\hline TG & 0.063 & 0.028 & 0.010 & 0.851 & 0.078 & 0.023 \\
\hline $\mathrm{HDL}-\mathrm{C}$ & 0.096 & 0.001 & 0.098 & 0.057 & 0.100 & 0.003 \\
\hline LDL-C & 0.195 & $<0.0001$ & 0.153 & 0.003 & 0.215 & $<0.0001$ \\
\hline \multicolumn{7}{|c|}{ Novel lipid markers } \\
\hline non-HDL-C & 0.180 & $<0.0001$ & 0.104 & 0.043 & 0.217 & $<0.0001$ \\
\hline $\operatorname{Lp}(\mathrm{a})$ & 0.062 & $<0.0001$ & 0.079 & 0.126 & 0.054 & 0.117 \\
\hline ApoA1 & 0.092 & 0.001 & 0.104 & 0.044 & 0.090 & 0.009 \\
\hline ApoB & 0.192 & $<0.0001$ & 0.138 & 0.007 & 0.213 & $<0.0001$ \\
\hline TRLP & 0.045 & 0.116 & 0.046 & 0.371 & 0.038 & 0.264 \\
\hline \multicolumn{7}{|c|}{ Derived lipid indicators } \\
\hline $\mathrm{TG} / \mathrm{HDL}-\mathrm{C}$ & 0.012 & 0.682 & -0.032 & 0.538 & 0.023 & 0.510 \\
\hline LDL-C/HDL-C & 0.073 & 0.010 & 0.026 & 0.620 & 0.089 & 0.009 \\
\hline $\mathrm{TC} / \mathrm{HDL}-\mathrm{C}$ & 0.037 & 0.195 & -0.019 & 0.712 & 0.056 & 0.102 \\
\hline ApoB/ApoA1 & 0.075 & 0.009 & 0.034 & 0.510 & 0.088 & 0.011 \\
\hline non-HDL-C/TC & 0.037 & 0.195 & -0.019 & 0.712 & 0.056 & 0.102 \\
\hline AIP & 0.012 & 0.682 & -0.032 & 0.538 & 0.023 & 0.510 \\
\hline $\mathrm{LCl}$ & 0.112 & $<0.0001$ & 0.042 & 0.414 & 0.139 & $<0.0001$ \\
\hline \multicolumn{7}{|c|}{ Other metabolic indexes } \\
\hline SBP & -0.014 & 0.632 & -0.001 & 0.988 & -0.033 & 0.343 \\
\hline $\mathrm{DBP}$ & -0.039 & 0.169 & -0.059 & 0.252 & -0.035 & 0.315 \\
\hline $\mathrm{BMI}$ & -0.007 & 0.815 & -0.042 & 0.417 & -0.008 & 0.818 \\
\hline Glucose & 0.104 & $<0.0001$ & 0.088 & 0.087 & 0.087 & 0.012 \\
\hline $\mathrm{HbA}_{1 \mathrm{C}}$ & 0.123 & $<0.0001$ & 0.111 & 0.032 & 0.098 & 0.004 \\
\hline $\mathrm{HGl}$ & 0.112 & $<0.0001$ & 0.076 & 0.141 & 0.085 & 0.013 \\
\hline TyG & 0.088 & 0.002 & 0.040 & 0.435 & 0.087 & 0.011 \\
\hline
\end{tabular}

PCSK9 proprotein convertase subtilisin/kexin type 9, CAD coronary artery disease, DM diabetes mellitus, non-DM non-diabetes mellitus, TC total cholesterol, TG triglyceride, $H D L-C$ high-density lipoprotein cholesterol, $L D L-C$ low-density lipoprotein cholesterol, non-HDL-C non-high-density lipoprotein cholesterol, $A p o A 1$ Apolipoprotein A1, $A p o B$ apolipoprotein B, TRLP triglyceride-rich lipoproteins, AIP atherogenic index of plasma, $L C I$ lipoprotein combine index, $S B P$ systolic blood pressure, $D B P$ diastolic blood pressure, $B M /$ body mass index, $H b A_{1 C}$ hemoglobin $A_{1 C}, H G I$ hemoglobin glycation index, TyG triglyceride glucose, $p<0.05$ suggests significant difference. (TRLP $=\mathrm{TC}-\mathrm{HDL}-\mathrm{C}-\mathrm{LDL}-\mathrm{C} ; \mathrm{LCl}=\mathrm{TC} * \mathrm{TG} * \mathrm{LDL} / \mathrm{HDL}-\mathrm{C} ; \mathrm{AIP}=\log (\mathrm{TG} / \mathrm{HDL}-\mathrm{C}) ; \mathrm{TyG}=\mathrm{Ln}(\mathrm{TG} * \mathrm{FPG} / 2) ; \mathrm{HGI}=\mathrm{observed} \mathrm{HbA} 1 \mathrm{c}-\mathrm{predicted} \mathrm{HbA} 1 \mathrm{c})$, $p<0.05$ suggests significant difference

\section{PCSK9, DM and cardiovascular outcomes}

Over a median of 3.3 years' follow-up, 103 patients developed subsequent MACEs (3 suffered nonfatal MI, 41 underwent coronary revascularization, 8 had ischemic stroke, 6 died and 45 experienced hospitalization because of unstable angina pectoris). High PCSK9 group had higher incidence of evens during follow-up time compared to low PCSK9 group [(67 (10.9\%) vs 36 (5.9\%), $p=0.001)$ ], while data from Additional file 1: Table S1, showed that DM group had higher prevalence of MACEs than non-DM group [50 (13.3\%) vs 50 $(6.3 \%), p<0.001]$. Kaplan Meier curve with the log-rank test displayed that patients in high PCSK9 group had lower event-free survival rate compared with those in low PCSK9 group ( $p=0.001$, Fig. $2 \mathrm{~b}$ ) and DM subjects were more likely to occur future worse cardiovascular events compared to patients without DM $(p<0.001$, Fig. 2a). Hereafter, participants were categorized into 4 subgroups when combined diabetic status with PCSK9 levels, those with high PCSK9 levels had significantly higher risk of MACEs than that with reference subgroup (non-DM plus low PCSK9 levels) despite of diabetic or nondiabetic population. DM plus high PCSK9 levels subgroup had the lowest cumulative event-free survival rate among the four subgroups $(p<0.001$, Fig. 2c). 


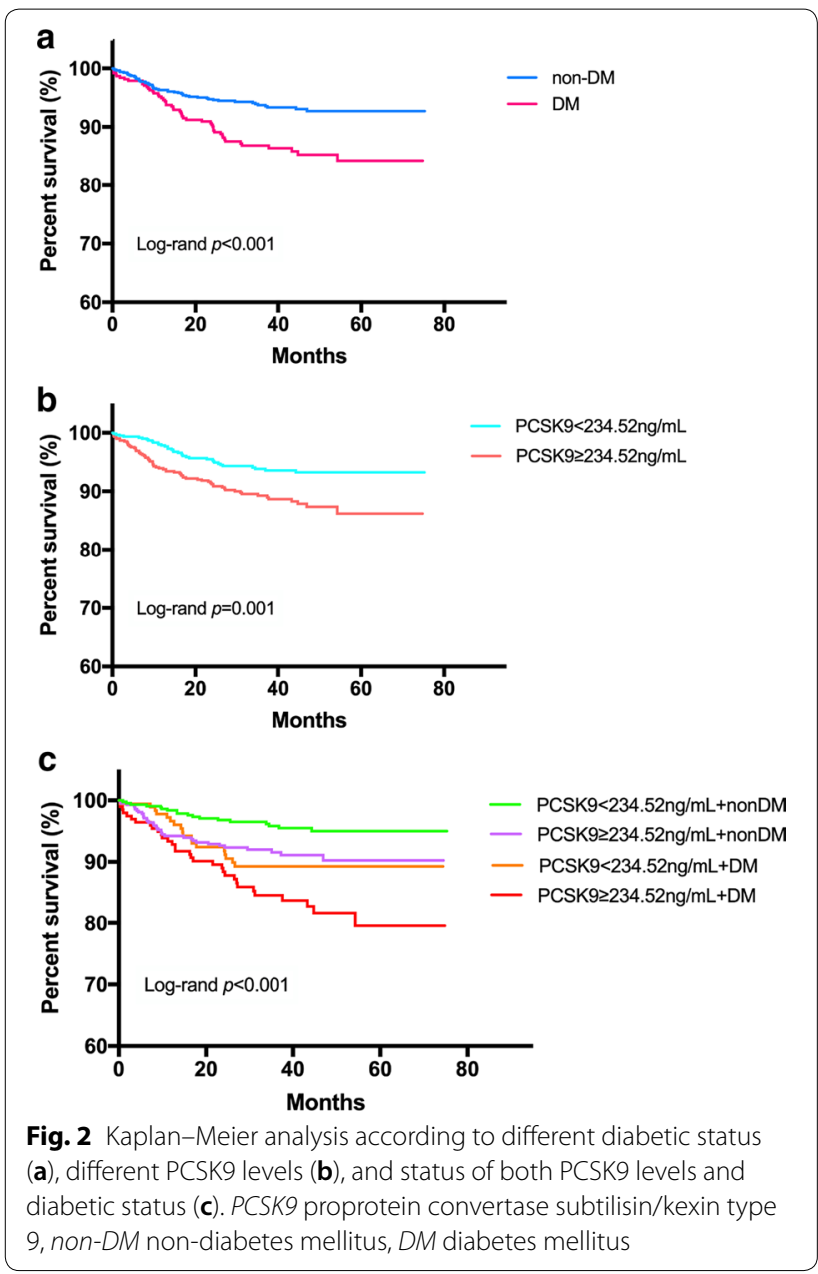

As presented in the Additional file 1: Table S2, univariate Cox regression analysis indicated that DM patients had 2.13-fold higher risk of MACEs than those without DM (HR: 2.130, 95\% CI: 1.447-3.135, $p<0.01$ ). After adjusting for confounding factors, the significant association still remained (Additional file 1: Table S2 and Fig. 3a). Meanwhile, the high PCSK9 group had significantly higher risk of MACEs than those in low PCSK9 group in this entire population (adjusted HR: 1.855, 95\% CI: $1.215-2.831, p<0.01$, Additional file 1: Table S2 and Fig. 3a). Notably, as a continuous variable, per 1-SD increase of PCSK9 level was significantly associated with 36.1 and $45.8 \%$ increased risk for MACEs in DM and non-DM groups, respectively (adjusted HR: 1.361, 95\% CI: $1.037-1.785, p<0.01$; adjusted HR: $1.458,95 \% \mathrm{CI}$ : $1.128-1.885, p<0.05$, respectively, Table 4 and Fig. $3 \mathrm{~b}$ ). Likewise, in the diabetic group, there was 2.294-fold (95\% CI: 1.230-4.278) higher risks of MACEs in patients with high PCSK than those with low PCSK9 after adjustment for established cardiovascular metabolic factors. Interestingly, multivariate Cox regression analysis based on the

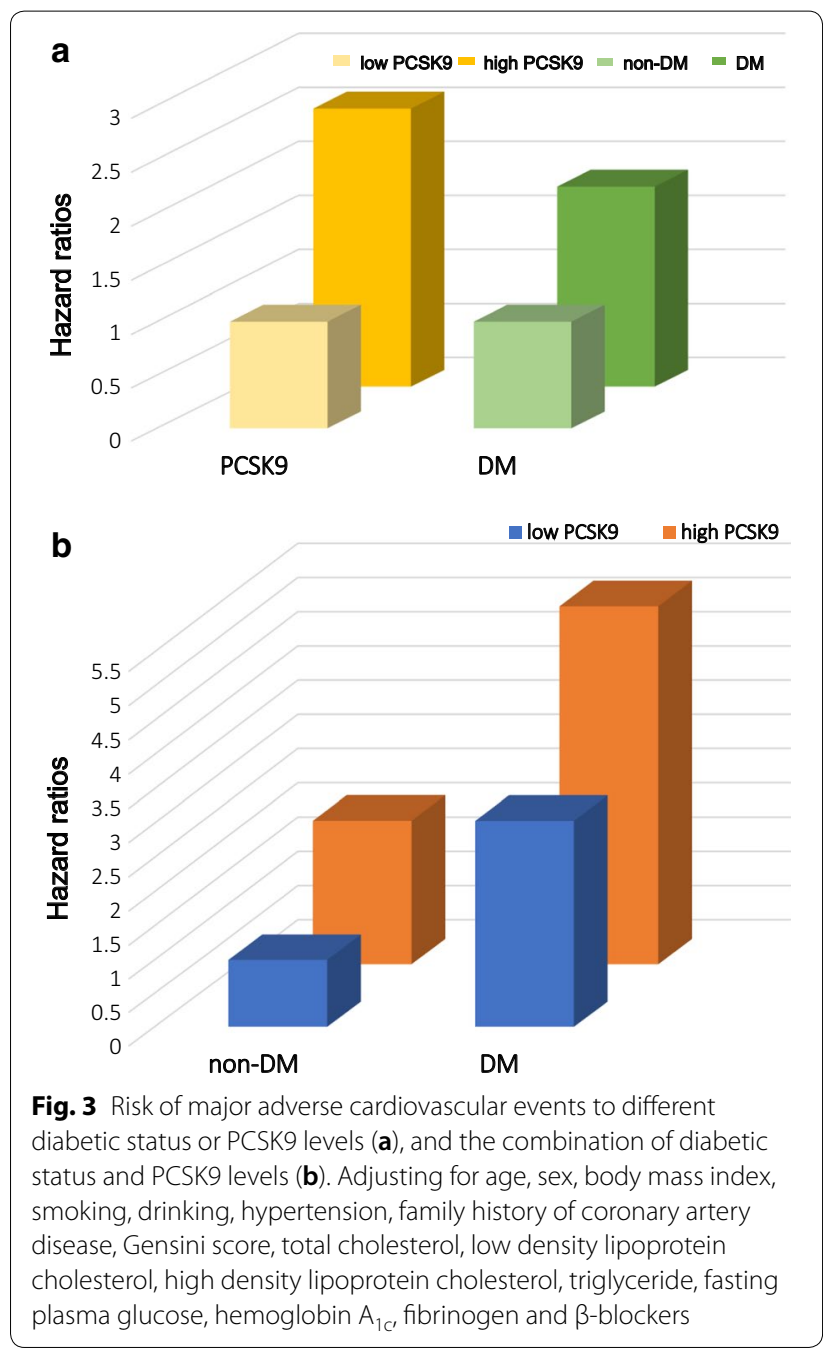

combination of diabetic status and PCSK9 levels revealed that patients in DM plus high PCSK9 levels, DM plus low PCSK9 levels and non-DM plus high PCSK9 levels groups had 5.233-fold (95\% CI: 2.546-10.757), 3.033-fold (95\% CI: 1.442-6.376) and 2.1-fold (95\% CI: 1.174-3.757) higher risk of MACEs (all $p<0.05$, Table 4 and Fig. $3 \mathrm{~b}$ ) than reference subgroup, respectively.

\section{Discussion}

In this study, we investigated the association of PCSK9 with CVMMs and the predictive value of PCSK9 for cardiovascular events in stable CAD patients with DM or without DM. Firstly, we found that plasma PCSK9 levels were significantly correlated with LCI and HGI, the novel CVMMs related to cardiovascular risks, in all CAD patients and positively associated with lipid- and glucoserelated CVMMs including TC, LDL-C, non-HDL-C and $\mathrm{HbA}_{1 \mathrm{C}}$ in DM participants with stable CAD. Besides, our results showed that circulating PCSK9 concentration at 
Table 4 PCSK9 levels in relation to MACEs in patients with different diabetic status

\begin{tabular}{|c|c|c|c|}
\hline \multirow[t]{2}{*}{ PCSK9 (ng/mL) } & \multicolumn{3}{|l|}{ HR $(95 \% \mathrm{Cl})$} \\
\hline & Crude model & Model 1 & Model 2 \\
\hline \multicolumn{4}{|l|}{ Non-DM } \\
\hline PCSK9 per-SD increase & $1.543(1.224-1.945)^{b}$ & $1.571(1.232-2.004)^{b}$ & $1.458(1.128-1.885)^{b}$ \\
\hline PCSK9 $<234.52 \mathrm{ng} / \mathrm{mL}$ & 1.0 (Reference) & 1.0 (Reference) & 1.0 (Reference) \\
\hline PCSK9 $\geq 234.52 \mathrm{ng} / \mathrm{mL}$ & $2.137(1.210-3.775)^{b}$ & $2.140(1.206-3.795)^{b}$ & $2.100(1.174-3.757)^{\mathrm{a}}$ \\
\hline \multicolumn{4}{|l|}{ DM } \\
\hline PCSK9 per-SD increase & $1.258(0.975-1.623)$ & $1.262(0.972-1.639)$ & $1.361(1.037-1.785)^{\mathrm{a}}$ \\
\hline PCSK9 $<234.52 \mathrm{ng} / \mathrm{mL}$ & $2.430(1.264-4.467)^{b}$ & $2.424(1.260-4.663)^{b}$ & $3.033(1.442-6.376)^{b}$ \\
\hline PCSK9 $\geq 234.52 \mathrm{ng} / \mathrm{mL}$ & $4.092(2.297-7.290)^{b}$ & $4.084(2.274-7.333)^{b}$ & $5.233(2.546-10.757)^{b}$ \\
\hline
\end{tabular}

baseline was positively associated with the risk of MACEs in diabetic patients with stable CAD even after adjusting for established cardiometabolic factors. Finally, data also indicated that the combination of high PCSK9 levels and DM had significantly higher cardiovascular events in our studied patients. To the best of our knowledge, this is the first study showing that PCSK9 can predict worse clinical cardiovascular outcomes in diabetic patients with stable CAD and the combination of plasma PCSK9 levels and diabetes status enhanced predictive value in CAD patients.

DM has become the one of major worldwide healthy issues and its prevalence has been rapidly growing in the past decades [28]. Of note, DM patients are more likely to have higher risk of cardiovascular events when accompanied by other metabolic disorders, especially in patients with ASCVD [1, 4]. Furthermore, atherogenic dyslipidemia has been reported to have an essential role in the prognosis of these two diseases, DM and ASCVD [29]. Even if receiving standard treatment, diabetic patients with CAD still have high cardiovascular risk. Therefore, further knowledge of cardiovascular risks related to diabetic patients with CAD will be helpful for better prevention and management for such kind of patients.

The best-known function of PCSK9 is posttranslational regulation of LDLR on hepatocytes to clear LDL-C from the circulation [30]. In recent years, it has been reported that PCSK9 is linked to atherosclerosis and become an attractive treatment target for CAD [10, 15, 27]. Meanwhile, PCSK9 levels were associated with several CVMMs which have been proposed as predictor of cardiovascular risks [18, 24-26]. However, the predictive role of PCSK9 on cardiovascular outcomes is currently controversial. A positive association with PCSK9 and cardiovascular events was found in the general population, in FH subjects, and in patients with acute coronary syndrome or stable CAD $[15,16,20,31]$. In contrast, a prospective cohort study of primary prevention included 716 initially healthy American women and displayed that the baseline plasma levels of PCSK9 cannot predict future cardiovascular events [32]. Moreover, the SURDIAGENE study found a similar result in DM population [33]. Hence, whether PCSK9 can be a novel predictor of prognosis and its correlation with metabolic factors need more investigations, especially in CAD patients with or without DM.

The associations of PCSK9 with cardiovascular metabolic parameters including lipid- and diabetes-related indicators, BMI and BP, have been found in different populations [17-19]. In patients with stable CAD, low plasma levels of PCSK9 were linked with a particular metabolic phenotype (low HDL-C, the metabolic syndrome, obesity, insulin resistance and DM) [19]. In CAD patients with DM who had high non-HDL-C/LDL-C levels, PCSK9 inhibitor significantly reduced atherogenic cholesterol and LDL-particle number versus control [34]. What's more, an animal study using obese-insulin resistant rat model, found that PCSK9 inhibition attenuated metabolic impairment and also ameliorated cardiovascular dysfunction more efficiently than atorvastatin [35]. In the current study, our results also showed a significant correlation between plasma PCSK9 levels and multiple CVMMs including traditional, novel and derived parameters in CAD patients with DM or without DM. Interestingly, in whole stable CAD patients, we found that PCSK9 was significantly and positively related to LCI and HGI, two novel lipid- or glycemic derived indicators and the potential cardiovascular outcomes predictors.[26, 36]. It 
was worth mentioning that, we did not find the association between PCSK9 and some indictors, mainly HDL-C, TG and their derived indexes in DM patients, which may be due to the higher heterogeneous in metabolic phenotypes. In addition, the present study showed that patients with high plasma PCSK9 concentration were more likely to be female, which was consistent with previous studies $[37,38]$. The reason for this phenomenon may be that plasma PCSK9 levels are influenced by hormones such as estrogen [39]. Besides, we found that low PCSK9 levels were associated with the feature of smoking and drinking was inconsistent with other studies [40, 41], which may need to investigate further in a large sample study.

In literature, the close relation between PCSK9 and DM has been documented. The results of available epidemiology and clinical studies showed a higher PCSK9 levels in diabetic patients $[17,18]$. Moreover, it is wellestablished that insulin resistance is the main cause of DM. Previous data suggested that insulin resistance and subsequent hyperinsulinemia could enhance PCSK9 expression and increased plasma levels of PCSK9 were related to poor glycemic control in DM [42]. Besides, it has been demonstrated that insulin resistance plays a pivotal role in PCSK9 homeostasis in severely obese patients [43]. PCSK9 critically controls LDLR expression in pancreas perhaps contributing to the maintenance of a proper physiological balance to limit cholesterol overload in beta cells [44]. Regarding pharmacology, it has been found that liraglutide can suppress PCSK9 expression through hepatocyte nuclear factor 1 alpha-dependent mechanism in HepG2 cells and $\mathrm{db} / \mathrm{db}$ mice [45]. In addition, as found above in this study, PCSK9 was significantly correlated with glycemic parameters including FPG and $\mathrm{HbA}_{1 \mathrm{C}}$. Meanwhile, our results indicated that PCSK9 or DM was independently predictors for MACEs respectively. The PCSK9 levels categorized according to its median and as a continuous variable were all independently related to poor outcomes even after adjustment for established cardiovascular risk factors in DM patients with CAD. Clearly, we not only analyzed the prognostic ability of PCSK9 but also further demonstrated the combined impact of elevated PCSK9 levels plus diabetic status. When added PCSK9 stratification and diabetic status to stratifying factors, patients in high PCSK9 group appeared to have the highest risk of subsequent cardiovascular events with DM (HR: 5.233 , 95\% CI: 2.546-10.757). These findings suggested that the measurement of PCSK9 might be useful to predict the occurrence of MACEs in diabetic patients with CAD and high PCSK9 levels plus DM could enhance the prediction of worse clinical outcomes.

The previous studies including ours have demonstrated that some lipid-lowering medications, such as statins, ezetimibe, can up-regulated the expression of PCSK9 genes and increased the circulating concentration of PCSK9 [46-48]. A meta-analysis of 15 clinical trials have demonstrated a significant increase in plasma PCSK9 levels after statin therapy, irrespective of the type of statin [49].Besides, recent data have indicated that PCSK9 inhibitors can significantly reduce circulating PCSK9 levels [48]. That is the reason why we chose the diabetic patients with stable CAD, who did not receive any lipidlowering therapy before admission as the participants to performed such study $[48,50]$.

Nevertheless, several limitations must be given consideration. First of all, the sample size and follow-up time of the current study were relatively small and short, which could lead to insufficient statistical power in analyses of subgroups. Moreover, due to fewer events, we could not further perform subgroup analyses for nonfatal MI, stroke and cardiovascular death. Secondly, we only measured the baseline of plasma PCSK9 levels and did not evaluate the effects of its longitudinal change (especially the effects of lipid-lowering drugs on PCSK9 levels after discharge) on cardiovascular events during follow-up time. Lastly, as with the feature of any observational and prospective study, only association but no causal link could be determined. Further prospective studies with larger sample size and long-term follow-up are required to confirm our findings.

\section{Conclusion}

In conclusion, our data, for the first time, showed that PCSK9 was associated with multiple CVMMs (including TC, LDL-C, non-HDL-C and $\mathrm{HbA}_{1 \mathrm{C}}$ ). Elevated PCSK9 levels were an independent predictor for adverse cardiovascular outcomes in diabetic patients with stable CAD and the combination of high PCSK9 and DM could increase predicting ability for cardiovascular events.

\section{Supplementary information}

Supplementary information accompanies this paper at https://doi. org/10.1186/s12933-020-01142-0.

Additional file 1: Table S1. Baseline characteristics of patients with DM and non-DM. Table S2. Cox regression analysis of MACEs according to different diabetic status and PCSK9 levels. Table S3. Therapy after admission of patients with events and without events.

\section{Abbreviations}

DM: Diabetes mellitus; CAD: Coronary artery disease; ASCVD: Atherosclerotic cardiovascular disease; LDL-C: Low-density lipoprotein cholesterol; PCSK9: Proprotein convertase subtilisin/kexin type 9; LDLR: Low-density lipoprotein cholesterol receptors; CVMMs: Cardiovascular metabolic markers; MACEs: Major adverse cardiovascular events; MI: Myocardial infarction; FH: Familial hypercholesterolemia; TG: Triglyceride; TC: Total cholesterol; HDL-C: Highdensity lipoprotein cholesterol; apo: Apolipoprotein; FPG: Fasting plasma glucose; $\mathrm{HbA}_{1}$ : Hemoglobin $\mathrm{A}_{1}$; BP: Blood pressure; PCl: Percutaneous coronary intervention; $\mathrm{CABG}$ : Coronary artery bypass grafting; BMI: Body mass 
index; non-HDL-C: Non-high-density lipoprotein cholesterol; LCl: Lipoprotein combine index; HGI: Hemoglobin glycation index; SD: Standard deviation; HR: Hazard ratios; $\mathrm{Cl}$ : Confidence intervals.

\section{Acknowledgements}

The authors thank all the staff and participants of this study for their important contributions.

\section{Authors' contributions}

PJ and LMM completed the project, analyzed the data, and wrote the manuscript. Li JJ designed the study, interpreted the data, and contributed to critically revising the article. XRX interpreted the data, and contributed to critically revising the article. JJL, CYX and GYL contributed to data collection. WNQ and ZCG contributed to recruitment of patients. DQ and SJ contributed to the collections of clinical data and procedure of laboratory examination. All authors read and approved the final manuscript.

\section{Funding}

This study was partly supported by Capital Health Development Fund (201614035) and Chinese Academy of Medical Sciences Innovation Fund for Medical Sciences (2016-I2M-1-011) awarded to Dr. Jian-Jun Li, MD, PhD.

\section{Availability of data and materials}

The datasets used and analyzed during the current study are available from the corresponding author on reasonable request.

\section{Ethics approval and consent to participate}

Approvals of the local ethics committees were obtained at all sites and the informed written consents were obtained from enrolled patients. This study complied with the Declaration of Helsinki and was approved by the hospital ethical review board (Fu Wai Hospital \& National Center for Cardiovascular Diseases, Beijing, China, approval number: 2013-442).

\section{Consent for publication}

Not applicable.

\section{Competing of interests}

The authors declared that they have no conflict of interest with respect to this manuscript.

\section{Received: 9 July 2020 Accepted: 27 September 2020}

Published online: 06 October 2020

\section{References}

1. Juutilainen A, Lehto S, Rönnemaa T, Pyörälä K, Laakso M. Type 2 diabetes as a "coronary heart disease equivalent": an 18-year prospective population-based study in Finnish subjects. Diabetes Care. 2005;28(12):2901-7.

2. Beckman JA, Creager MA, Libby P. Diabetes and atherosclerosis: epidemiology, pathophysiology, and management. JAMA. 2002;287(19):2570-81.

3. Haffner SM, Lehto S, Rönnemaa T, Pyörälä K, Laakso M. Mortality from coronary heart disease in subjects with type 2 diabetes and in nondiabetic subjects with and without prior myocardial infarction. N Engl J Med. 1998;339(4):229-34.

4. Newman JD, Schwartzbard AZ, Weintraub HS, Goldberg IJ, Berger JS. Primary Prevention of Cardiovascular Disease in Diabetes Mellitus. J Am Coll Cardiol. 2017;70(7):883-93.

5. Colhoun HM, Betteridge DJ, Durrington PN, Hitman GA, Neil HAW, Livingstone SJ, Thomason MJ, Mackness MI, Charlton-Menys V, Fuller JH. Primary prevention of cardiovascular disease with atorvastatin in type 2 diabetes in the Collaborative Atorvastatin Diabetes Study (CARDS): multicentre randomised placebo-controlled trial. Lancet. 2004;364(9435):685-96.

6. Kereiakes DJ, Robinson JG, Cannon CP, Lorenzato C, Pordy R, Chaudhari U, Colhoun HM. Efficacy and safety of the proprotein convertase subtilisin/ kexin type 9 inhibitor alirocumab among high cardiovascular risk patients on maximally tolerated statin therapy: The ODYSSEY COMBO I study. Am Heart J. 2015;169(6):906

7. Fruchart JC, Santos RD, Aguilar-Salinas C, Aikawa M, Al Rasadi K, Amarenco P, Barter PJ, Ceska R, Corsini A, Després JP, et al. The selective peroxisome proliferator-activated receptor alpha modulator (SPPARMa) paradigm: conceptual framework and therapeutic potential: a consensus statement from the International Atherosclerosis Society (IAS) and the Residual Risk Reduction Initiative (R3i) Foundation. Cardiovasc Diabetol. 2019;18(1):71.

8. Seidah NG, Awan Z, Chrétien M, Mbikay M. PCSK9: a key modulator of cardiovascular health. Circ Res. 2014;114(6):1022-36.

9. Cunningham D, Danley DE, Geoghegan KF, Griffor MC, Hawkins JL, Subashi TA, Varghese AH, Ammirati MJ, Culp JS, Hoth LR, et al. Structural and biophysical studies of PCSK9 and its mutants linked to familial hypercholesterolemia. Nat Struct Mol Biol. 2007;14(5):413-9.

10. Cesaro A, Bianconi V, Gragnano F, Moscarella E, Fimiani F, Monda E, Scudiero O, Limongelli G, Pirro M, Calabrò P. Beyond cholesterol metabolism: The pleiotropic effects of proprotein convertase subtilisin/kexin type 9 (PCSK9). Genetics, mutations, expression, and perspective for long-term inhibition. BioFactors. 2020;46:1-14.

11. Ferri N, Marchianò S, Tibolla G, Baetta R, Dhyani A, Ruscica M, Uboldi P, Catapano AL, Corsini A. PCSK9 knock-out mice are protected from neointimal formation in response to perivascular carotid collar placement. Atherosclerosis. 2016:253:214-24.

12. Camera M, Rossetti L, Barbieri SS, Zanotti I, Canciani B, Trabattoni D, Ruscica M, Tremoli E, Ferri N. PCSK9 as a Positive Modulator of Platelet Activation. J Am Coll Cardiol. 2018;71(8):952-4.

13. Bonaca MP, Nault P, Giugliano RP, Keech AC, Pineda AL, Kanevsky E, Kuder J, Murphy SA, Jukema JW, Lewis BS, et al. Low-density lipoprotein cholesterol lowering with evolocumab and outcomes in patients with peripheral artery disease: insights from the Fourier Trial (Further Cardiovascular Outcomes Research With PCSK9 Inhibition in Subjects With Elevated Risk). Circulation. 2018;137(4):338-50.

14. Szarek M, White HD, Schwartz GG, Alings M, Bhatt DL, Bittner VA, Chiang C-E, Diaz R, Edelberg JM, Goodman SG, et al. Alirocumab reduces total nonfatal cardiovascular and fatal events: the ODYSSEY OUTCOMES trial. J Am Coll Cardiol. 2019;73(4):387-96.

15. Leander K, Mälarstig A, Van't Hooft FM, Hyde C, Hellénius M-L, Troutt JS, Konrad RJ, Öhrvik J, Hamsten A, de Faire U. Circulating proprotein convertase subtilisin/kexin type 9 (PCSK9) predicts future risk of cardiovascular events independently of established risk factors. Circulation. 2016;133(13):1230-9.

16. Li J-J, Li S, Zhang Y, Xu R-X, Guo Y-L, Zhu C-G, Wu N-Q, Qing P, Gao Y, Sun , et al. Proprotein convertase subtilisin/kexin type 9, c-reactive protein, coronary severity, and outcomes in patients with stable coronary artery disease: a Prospective Observational Cohort Study. Medicine (Baltimore). 2015;94(52):e2426.

17. Levenson AE, Shah AS, Khoury PR, Kimball TR, Urbina EM, de Ferranti SD, Maahs DM, Dolan LM, Wadwa RP, Biddinger SB. Obesity and type 2 diabetes are associated with elevated PCSK9 levels in young women. Pediatr Diabetes. 2017;18(8):755-60.

18. Yang S-H, Li S, Zhang Y, Xu R-X, Guo Y-L, Zhu C-G, Wu N-Q, Cui C-J, Sun J, Li J-J. Positive correlation of plasma PCSK9 levels with HbA1c in patients with type 2 diabetes. Diabetes Metab Res Rev. 2016;32(2):193-9.

19. Caselli C, Del Turco S, Ragusa R, Lorenzoni V, De Graaf M, Basta G, Scholte A, De Caterina R, Neglia D. Association of PCSK9 plasma levels with metabolic patterns and coronary atherosclerosis in patients with stable angina. Cardiovasc Diabetol. 2019;18(1):144.

20. Cao Y-X, Jin J-L, Sun D, Liu H-H, Guo Y-L, Wu N-Q, Xu R-X, Zhu C-G, Dong Q, Sun J, et al. Circulating PCSK9 and cardiovascular events in FH patients with standard lipid-lowering therapy. J Transl Med. 2019;17(1):367.

21. Cao YX, Liu HH, Jin JL, Sun D, Guo YL, Wu NQ, Zhu CG, Xu RX, Sun J, Santos $\mathrm{RD}$, et al. Plasma proprotein convertase subtilisin/kexin type 9 concentration and recurrent cardiovascular events in patients with familial hypercholesterolemia. Eur J Prev Cardiol. 2019. https://doi.org/10.1177/20474 87319880985.

22. Cao Y-X, Liu H-H, Sun D, Jin J-L, Xu R-X, Guo Y-L, Wu N-Q, Zhu C-G, Li S, Zhang Y, et al. The different relations of PCSK9 and Lp(a) to the presence and severity of atherosclerotic lesions in patients with familial hypercholesterolemia. Atherosclerosis. 2018. https://doi.org/10.1016/j.atheroscle rosis.2018.07.030

23. Lamprea-Montealegre JA, Staplin N, Herrington WG, Haynes R, Emberson J, Baigent C, de Boer $\mathrm{H}$. Apolipoprotein B, triglyceride-rich lipoproteins, and risk of cardiovascular events in persons with CKD. Clin J Am Soc Nephrol. 2020;15(1):47-60. 
24. Wu T-T, Gao Y, Zheng Y-Y, Ma Y-T, Xie X. Atherogenic index of plasma (AIP): a novel predictive indicator for the coronary artery disease in postmenopausal women. Lipids Health Dis. 2018;17(1):197.

25. Hempe JM, Liu S, Myers L, McCarter RJ, Buse JB, Fonseca V. The hemoglobin glycation index identifies subpopulations with harms or benefits from intensive treatment in the ACCORD trial. Diabetes Care. 2015;38(6):1067-74

26. Jin J-L, Sun D, Cao Y-X, Guo Y-L, Wu N-Q, Zhu C-G, Gao Y, Dong Q-T, Zhang H-W, Liu G, et al. Triglyceride glucose and haemoglobin glycation index for predicting outcomes in diabetes patients with new-onset, stable coronary artery disease: a nested case-control study. Ann Med. 2018;50(7):576-86.

27. Li S, Zhang Y, Xu R-X, Guo Y-L, Zhu C-G, Wu N-Q, Qing P, Liu G, Dong Q, Li $J$-J. Proprotein convertase subtilisin-kexin type 9 as a biomarker for the severity of coronary artery disease. Ann Med. 2015;47(5):386-93.

28. Ng LC, Gupta M. Transdermal drug delivery systems in diabetes management: a review. Asian J Pharm Sci. 2020;15(1):13-25.

29. Stone NJ, Robinson JG, Lichtenstein AH, Bairey Merz CN, Blum CB, Eckel RH, Goldberg AC, Gordon D, Levy D, Lloyd-Jones DM, et al. 2013 ACC/ AHA guideline on the treatment of blood cholesterol to reduce atherosclerotic cardiovascular risk in adults: a report of the American College of Cardiology/American Heart Association Task Force on Practice Guidelines. Circulation. 2014;129(25 Suppl 2):S1-45.

30. Lambert G, Sjouke B, Choque B, Kastelein JJP, Hovingh GK. The PCSK9 decade. J Lipid Res. 2012;53(12):2515-24.

31. Gencer B, Montecucco F, Nanchen D, Carbone F, Klingenberg R, Vuilleumier N, Aghlmandi S, Heg D, Räber L, Auer R, et al. Prognostic value of PCSK9 levels in patients with acute coronary syndromes. Eur Heart J. 2016;37(6):546-53.

32. Ridker PM, Rifai N, Bradwin G, Rose L. Plasma proprotein convertase subtilisin/kexin type 9 levels and the risk of first cardiovascular events. Eur Heart J. 2016;37(6):554-60.

33. El Khoury P, Roussel R, Fumeron F, Abou-Khalil Y, Velho G, Mohammedi K, Jacob MP, Steg PG, Potier L, Ghaleb Y, et al. Plasma proprotein-convertase-subtilisin/kexin type 9 (PCSK9) and cardiovascular events in type 2 diabetes. Diabetes Obes Metab. 2018;20(4):943-53.

34. Ray KK, Del Prato S, Müller-Wieland D, Cariou B, Colhoun HM, Tinahones FJ, Domenger C, Letierce A, Mandel J, Samuel R, et al. Alirocumab therapy in individuals with type 2 diabetes mellitus and atherosclerotic cardiovascular disease: analysis of the ODYSSEY DM-DYSLIPIDEMIA and DM-INSULIN studies. Cardiovasc Diabetol. 2019;18(1):149.

35. Amput P, Palee S, Arunsak B, Pratchayasakul W, Kerdphoo S, Jaiwongkam T, Chattipakorn SC, Chattipakorn N. PCSK9 inhibitor effectively attenuates cardiometabolic impairment in obese-insulin resistant rats. Eur J Pharmacol. 2020;883:173347.

36. Oguntola SO, Hassan MO, Duarte R, Dix-Peek T, Dickens C, Olorunfemi G, Vachiat A, Paget G, Manga P, Naicker S. Atherosclerotic vascular disease and its correlates in stable black South African kidney transplant recipients. Int J Nephrol Renovasc Dis. 2018;11:187-93.

37. Zhang Y, Zhu C-G, Xu R-X, Li S, Guo Y-L, Sun J, Li J-J. Relation of circulating PCSK9 concentration to fibrinogen in patients with stable coronary artery disease. J Clin Lipidol. 2014;8(5):494-500.
38. Zhang Z, Wei TF, Zhao B, Yin Z, Shi QX, Liu PL, Liu LF, Liu L, Zhao JT, Mao S, et al. Sex Differences associated with circulating PCSK9 in patients presenting with acute myocardial infarction. Sci Rep. 2019;9(1):3113.

39. Ghosh M, Gälman C, Rudling M, Angelin B. Influence of physiological changes in endogenous estrogen on circulating PCSK9 and LDL cholesterol. J Lipid Res. 2015;56(2):463-9.

40. Gencer B, Pagano S, Vuilleumier N, Satta N, Delhumeau-Cartier C, Meier C, Bavamian S, Montecucco F, Mach F, Calmy A. Clinical, behavioral and biomarker predictors of PCSK9 levels in HIV-infected patients naïve of statin therapy: A cross-sectional analysis from the Swiss HIV cohort. Atherosclerosis. 2019;284:253-9.

41. Lee JS, Rosoff D, Luo A, Longley M, Phillips M, Charlet K, Muench C, Jung J, Lohoff FW. PCSK9 is increased in cerebrospinal fluid of individuals with alcohol use disorder. Alcohol Clin Exp Res. 2019;43(6):1163-9.

42. Miao J, Manthena PV, Haas ME, Ling AV, Shin D-J, Graham MJ, Crooke RM, Liu J, Biddinger SB. Role of insulin in the regulation of proprotein convertase subtilisin/kexin type 9. Arterioscler Thromb Vasc Biol. 2015;35(7):1589-96.

43. Zenti MG, Lupo MG, De Martin S, Altomari A, Galvan S, Aventaggiato M, Maneschi C, Sandri D, Paiola E, Battistoni M, et al. Impact of bariatric surgery-induced weight loss on circulating PCSK9 levels in obese patients. Nutr Metab and Cardiovasc Dis. 2020. https://doi.org/10.1016/j. numecd.2020.07.013.

44. Da Dalt L, Ruscica M, Bonacina F, Balzarotti G, Dhyani A, Di Cairano E, Baragetti A, Arnaboldi L, De Metrio S, Pellegatta F, et al. PCSK9 deficiency reduces insulin secretion and promotes glucose intolerance: the role of the low-density lipoprotein receptor. Eur Heart J. 2019:40(4):357-68.

45. Yang SH, Xu RX, Cui CJ, Wang Y, Du Y, Chen ZG, Yao YH, Ma CY, Zhu CG, Guo YL, et al. Liraglutide downregulates hepatic LDL receptor and PCSK9 expression in HepG2 cells and db/db mice through a HNF-1a dependent mechanism. Cardiovasc Diabetol. 2018;17(1):48.

46. Glerup S, Schulz R, Laufs U, Schluter KD. Physiological and therapeutic regulation of PCSK9 activity in cardiovascular disease. Basic Res Cardiol. 2017;112(3):32.

47. Guo Y-L, Liu J, Xu R-X, Zhu C-G, Wu N-Q, Jiang L-X, Li J-J. Short-term impact of low-dose atorvastatin on serum proprotein convertase subtilisin/kexin type 9. Clin Drug Investig. 2013;33(12):877-83.

48. Macchi C, Banach M, Corsini A, Sirtori CR, Ferri N, Ruscica M. Changes in circulating pro-protein convertase subtilisin/kexin type 9 levels - experimental and clinical approaches with lipid-lowering agents. Eur J Prev Cardiol. 2019;26(9):930-49.

49. Sahebkar A, Simental-Mendía LE, Guerrero-Romero F, Golledge J, Watts GF. Effect of statin therapy on plasma proprotein convertase subtilisin kexin 9 (PCSK9) concentrations: a systematic review and meta-analysis of clinical trials. Diabetes Obes Metab. 2015;17(11):1042-55.

50. Cui CJ, Li S, Li JJ. PCSK9 and its modulation. Clin Chim Acta. 2015:440:79-86.

\section{Publisher's Note}

Springer Nature remains neutral with regard to jurisdictional claims in published maps and institutional affiliations.
Ready to submit your research? Choose BMC and benefit from:

- fast, convenient online submission

- thorough peer review by experienced researchers in your field

- rapid publication on acceptance

- support for research data, including large and complex data types

- gold Open Access which fosters wider collaboration and increased citations

- maximum visibility for your research: over 100M website views per year

At BMC, research is always in progress.

Learn more biomedcentral.com/submissions 